\title{
NOTES ET INFORMATIONS
}

\section{Hyperinfestation d'Ecureuils (Sciurus vulgaris) par un Anoploure, Neohema- topinus sciuri Jancke, 1931.}

Le 7 janvier 1966, un écureuil fut trouvé mort à St-Odilïenberg (province du Limbourg, Hollande). Dans les environs de cette localité, un grand nombre d'autres écureuils mouraient également vers la même époque. Il semblait s'agir pour l'ensemble de ces cas, d'après le collecteur de ce matériel, de morts provoquées par la présence d'un grand nombre d'ectoparasites.

L'écureuil nous fut envoyé (1) pour identification de ses parasites, l'hôte entier étant placé en liquide conservateur.

En dehors de quelques exemplaires (2) d'une puce banale, spécifique de l'écureuil (Monopsyllus sciurorum sciurorum Schrank, 1857), nous avons eu la surprise de récolter une quantité extraordinaire d'un pou, Neohematopinus sciuri Jancke 1931, pou également inféodé d'ailleurs à l'écureuil. Après un épouillage à la pince, puis à la brosse, de plusieurs heures, devant le nombre très élevé de parasites restant encore sur l'animal, nous avons été amené à faire une estimation du nombre total, en dénombrant, à la loupe binoculaire, les poux encore accrochés à la peau, sur quelques surfaces bien délimitées et mesurées, choisies de façon à obtenir des chiffres moyens, en tenant compte du fait que la répartition n'est pas homogène sur l'hôte. Finalement, après évaluation de la surface corporelle de cet écureuil, nous sommes arrivé au total, approximatif certes, mais effarant, de 80000 poux, ce qui représente, au centimètre carré, une densité de plus de 300 Insectes. Précisons que dans ce chiffre figurent tous les stades (larves et adultes), à l'exclusion des œufs qui ne furent pas dénombrés.

Bien que plusieurs autres Neohematopinus soient connus comme vecteurs d'épizooties [Pasteurella pestis transmis par N. citelli, P. tularense par $N$. laeviusculus, Borrelia sp. (hispanica ?) par $N$. pectinifer], nous pensons que l'action spoliatrice et toxique propre de cette masse de parasites est amplement suffisante pour expliquer la mort des écureuils.

Il resterait toutefois à expliquer la pullulation de ces poux dans cette région du Limbourg. Nous n'avions jamais noté sur des animaux de taille identique des nombres d'Anoploures supérieurs à 100 , la moyenne étant aux environs de 15 par animal parasité. De plus, l'époque où fut notée cette massive prolifération correspond, au moins pour les Anoploures européens parasites d'autres Rongeurs étudiés jusqu'à présent, à la période de plus faible densité.

Notons pour terminer que l'examen macroscopique de la cavité générale et du tube digestif n'a permis de déceler aucun endoparasite. L'estomac et l'ensemble de l'intestin étaient vides, sans la moindre trace de matières en transit.

\section{J.-C. Beaucournu.}

(Laboratoire de Parasitologie et Zoologie de la Faculté de Médecine et de Pharmacie de Rennes Prof. J.-M. Dову).

(1) Cet écureuil, collecté par A.J. Verbeek, nous fut confié pour étude par le Prof. Van Bree (Zoological Museum, Amsterdam) à qui nous adressons nos très vifs remerciements.

(2) $1 \approx, 2 \%$. Ce nombre n’a pas de signification, car la plupart des puces ont dû quitter l'hôte soit à sa mort, soit avant, devant la prolifération des Anoploures. 